\title{
NEW INITIATIVES IN ASTRONOMICAL FACILITIES IN CHINA
}

\author{
LI QIBIN \\ Beijing Astronomical Observatory, \\ Beijing, 100080 China, P.R.
}

Proposals on future astronomy facilities have been surveyed in China recent years. Among the others, the Large Multi-Object Spectroscopy Telescope (LAMOST) has been cosidered to have higher priority by the astronomical community because of its optimum fit of large aperture and large field of view (FOV) in the design. The LAMOST has been approved as a national project this year.

The LAMOST is designed as a reflecting Schmidt telescope. The optical system consists of a $6 \mathrm{~m} \times 6.7 \mathrm{~m}$ spherical primary mirror and a $4.4 \mathrm{~m} \times 5.7 \mathrm{~m}$ aspheric secondary mirrior which lies at the center of the sphere $40 \mathrm{~m}$ away. The light from stars and galaxies is reflected to the primary by the secondary and then to a focus between the two mirriors. The aspheric shape of the secondary mirrior is maintained with the help of active optics to eliminate spherical aberration in real time. The primary and the secondary respectively consist of 37 and 24 seperately hexagonal segments with diagonal lengths of $1.1 \mathrm{~m}$. The effective aperture is $4 \mathrm{~m}$. The focal length is $20 \mathrm{~m}$. The FOV is about 5 degrees giving a linear size of $1.75 \mathrm{~m}$. Due to the large FOV, about 4000 fibers can be attached to the focal surface so that spectra of 4000 objects can be simultaneously observed.

The primary mirror facing forward to north is fixed on the foundation. The aspheric secondary mirror alt-azimuthally mounted also plays the role of siderostat. The light axis of the optical systern is inclined 25 degrees to the horizon. The telescope tracks celestial objects for 1 hour to 1.5 hours before and after passing through the meridian.

In general, the larger the aperture of telescope is, the smaller the FOV is. Considering most current approaches on larger aperture telescopes, such as KECK, VLT, SUBARU, GEMINI, COLUMBUS, HET, SALT etc, Chinese astronomers hope to contribute to wide field astronomy by their special design of a facility that is larger both in aperture and in field of view.

The government will fund the cost of LAMOST from the end of this year. The LAMOST project is scheduled to be completed in seven years. The telescope will be installed in the Xinglong Observing Station of the Beijing Astronomical Observatory.

Besides the ground-based facility, a Space Solar Telescope (SST) has been proposed by our colleagues. The design of SST is based on a space equivalent of the multi-channel magnetic field solar telescope in the Huairou Solar Observing Station of the Beijing Astronomical Observatory. The SST will be a $1 \mathrm{~m}$ telescope with two -dimensional real time polarizing spectrography. The aim of SST is to explore the three dimensional structure of the solar vector magnetic field and velocity field with 0.1 arcsec. resolution, and the fine evolution of solar active regions. The satellite will weigh 2.5 tons. The data-transmitting rate will be about half a gigabyte per day. Techniques used in SST will be very complicated so we begin with a balloon solar telescope as the first stage and technological test. The balloon-borne telescope will be launched this autumn. The phase A study for the SST has almost completed. The SST should be addressed in the national space programme.

Chinese radio astronomers are keen on the LT project, the next generation facility for radio astronomy. This international project was proposed at the 24th Assembly of URSI in Kyoto in 1993. Astronomers from 10 countries come to the LT Working Group. The sum of the receiving area of LT is $1 \mathrm{~km}$ square, in which single dish aperature is $300-400 \mathrm{~m}$. A LTWG meeting was held in China just three weeks ago. The LTWG astronomers seem to be interested in finding an observing site in China. In Guizhou Province, some karst sites are likely suitable to build Arecibo-type radio telescope. The radio astronomers have been actively promoting the LT proposal.

$$
890
$$




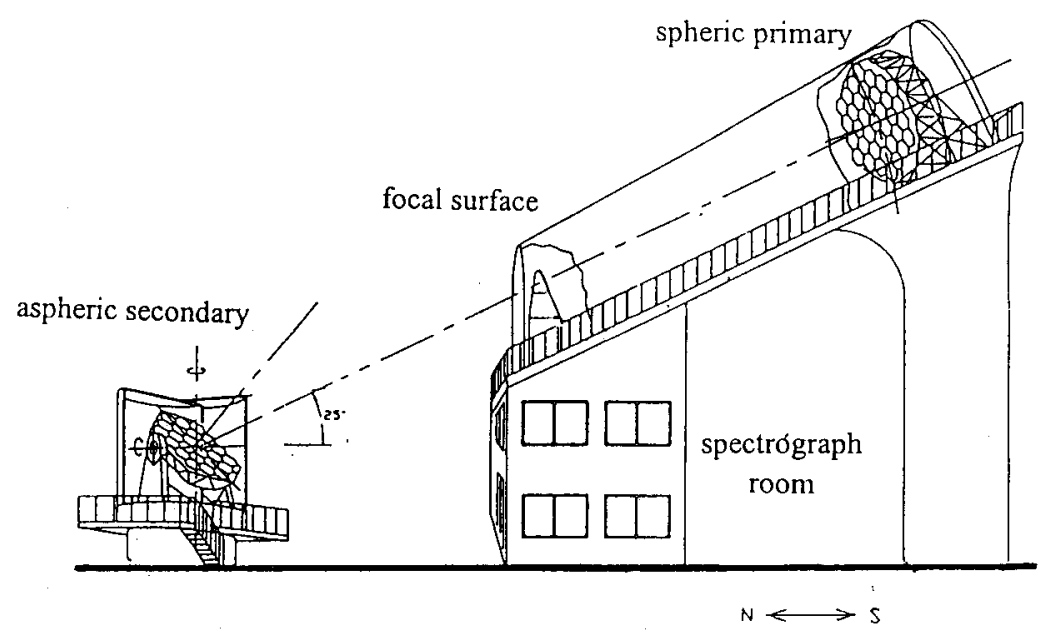

Figure 1. Schematic View of LAMOST 